\title{
Diferencias individuales como moderadoras de la relación estrés-salud en docentes universitarios
}

\section{Individual differences as moderators of the relationship between stress and health in university teachers}

\author{
Alba Lousinha ${ }^{1}$ \\ Instituto Universitario de Tecnología Dr. Federico Rivero Palacio, Caracas, Venezuela \\ Leticia Guarino ${ }^{2}$ \\ Universidad Simón Bolívar, Caracas, Venezuela \\ (Rec: noviembre 2012 - Acep: febrero 2013)
}

\begin{abstract}
Resumen
La presente investigación tuvo como objetivo estudiar el papel moderador de ciertas diferencias individuales como la Sensibilidad Emocional, la Tolerancia a la Incertidumbre y los Estilos de Afrontamiento en la relación estréssalud de docentes universitarios. Para ello, una muestra de 211 docentes pertenecientes a distintas instituciones públicas de educación superior de la ciudad de Caracas-Venezuela, completó instrumentos de auto-reporte de las variables estudiadas, siendo los datos sometidos a análisis de regresión jerárquica para determinar el efecto moderador de las diferencias individuales. Los resultados revelaron que el estilo de afrontamiento emocional y la sensibilidad egocéntrica negativa actuaron como moderadoras de la relación entre el estrés y la salud percibida de los profesores, específicamente como factores de riesgo en el proceso, confirmando el modelo de moderación para estas variables establecido en estudios previos.
\end{abstract}

Palabras clave: diferencias individuales, estrés, docentes universitarios.

\begin{abstract}
The aim of the present study was to determine the moderating role of certain individual differences, such as Emotional Sensitivity, Tolerance to Uncertainty, and Coping Styles, into the relationship between stress and health in university teachers. To do so, a sample of 211 teachers belonging to different public institutions of higher education from Caracas-Venezuela completed self-report instruments related to the studied variables. Data was subsequently analyzed using hierarchical regression equations to determine the moderating role of the mentioned individual differences. Results revealed that the Emotional Coping Style and the Negative Egocentric Sensitivity acted as moderators into the relationship between stress and perceived health of the participant teachers, specifically acting as risk factors of the process, and confirming the moderating model of these variables previously established in other studies.
\end{abstract}

Key words: individual differences, stress, university teachers

\footnotetext{
1 Dpto. de Extensión. Edificio Sede, piso 2. Instituto Universitario de Tecnología Dr. Federico Rivero Palacio Km. 8, Carretera Panamericana vía Los Teques. Caracas - Venezuela Telf. 0058021268284 66. E-mail: alba.lousinha@gmail.com.

2 Dpto. de Ciencia y Tecnología del Comportamiento. Edificio de Estudios Generales, 1er. Piso. Valle de Sartenejas, Aptdo. Postal 89.000 - A. Edo. Miranda. Venezuela. Tel.: 00582129063595 Fax: 0058212906 3811. E-mail: lguarino@usb.ve.
} 


\section{Introducción}

Junto a corredores de bolsa, gerentes en cargos estratégicos o mineros, se suele citar a profesionales con perfil asistencial: médicos, enfermeros, psicólogos, clérigos, y especialmente a los docentes, como trabajadores mayormente expuestos a situaciones generadoras de tensión (Guil, 1998; Guerrero, 2003; Cisneros Blas \& Ramírez Sandoval, 2009). También es conocido que el impacto de los sucesos ambientales varía en concordancia con las diferencias individuales, en otras palabras, varía según los grados particulares de vulnerabilidad, la tolerancia ante situaciones confusas y el modo personal de evaluar los propios recursos al afrontar (Lazarus \& Folkman, 1986; Guarino, 2009); por ende es razonable suponer que los docentes universitarios no escapen de esta realidad. La percepción de un evento como estresante o no, la frecuencia de situaciones vitales difíciles como perder el empleo, enfermarse seriamente, etc., o la tendencia a suspender clases reportando mayor nivel de malestar, pueden ser el producto esperable para un individuo con unas características de personalidad determinadas, de tal suerte que su salud y bienestar en términos generales estén íntimamente ligados a factores psicológicos.

En estudios previos se ha comprobado la relación entre estrés psicológico y diversas enfermedades como problemas cardiovasculares y cáncer (Baum \& Posluszny, 1999; Pamdya, 1998; Stahl \& Hauger, 1994; Tennant, 2000, en Ng \& Jeffery, 2003; Cohen, JanickiDeverts \& Miller, 2007). Una explicación tentativa de tal asociación consiste en que el estrés promueve comportamientos poco saludables que se convierten en factores de riesgo, como tabaquismo, consumo de alcohol e ingesta de grasas, entre otras (Guarino, 2012; Steptoe, 1991). El nexo conceptual que vincula los comportamientos poco saludables y el estrés, es el manejo inadecuado que la persona hace de su estado anímico. Dicho de manera simple, estados aversivos pueden conducir a la gente a manifestar conductas insanas (Zillman \& Bryant, 1985, en Ng \& Jeffery, 2003). En concordancia con estos planteamientos teóricos, cabe esperar una relación entre variables de tipo emociona; el modo de evaluar y responder a situaciones estresantes; y la forma de percibir los propios estados de salud.

Particularmente en lo concerniente a la profesión docente, las propias actividades de instrucción y formación se constituyen en un reto que demanda del profesor universitario entregarse, actualizarse e innovar estrategias continuamente. En un artículo centrado sobre el estrés del profesor universitario, Tomé y Urdiales (1996) exponen que con frecuencia estos profesionales llamados 'asistenciales', con una filosofía de trabajo de implicación y compromiso personal, son más propensos a sufrir de estrés. Por una parte manifiestan altas expectativas personales de eficiencia y entrega, por otra parte se enfrentan a las limitaciones de pocos recursos institucionales o baja motivación extrínseca. El conflicto entre expectativas y realidad trae como consecuencia una respuesta displacentera, producto del desequilibrio entre aspiraciones y resultados, sobre todo si estos últimos son percibidos como amenazantes de la imagen o de la competencia profesional. Más adelante, estas autoras sostienen que algunas de las manifestaciones comunes de tal conflicto son: la ansiedad, la frustración, la desilusión, el abandono, el desinterés y la depresión; de modo que si tal disfunción emocional se prolonga puede traer repercusiones en la salud, el bienestar y la eficiencia.

En otros trabajos sobre el contexto profesional y las variables de personalidad en la labor docente en países Iberoamericanos, se reportan altos porcentajes de decepción y malestar entre los profesores, incluso en los más jóvenes, quienes atribuyen su desánimo a la desvalorización de la profesión, la pérdida de estatus, la falta de apoyo, la carencia de recursos, las dificultades crecientes en la relación con alumnos, pares y autoridades administrativas. Todo ello. A pesar de tener un cargo relativamente estable en tiempos de crisis económica (García, 1996; González, 2008; Sánchez $\&$ Maldonado, 2003).

En tal sentido, parece lógico pensar que el poseer una capacidad para responder ante la incertidumbre generada por los imprevistos, especialmente los relacionados con la función educativa, denota buen manejo emocional, adecuados procesos cognitivos y favorece la adaptabilidad frente a los cambios; contribuyendo a disminuir el estrés. En tanto que se prevé que la intolerancia a la incertidumbre, por implicar la percepción de eventos ambiguos como posibles amenazas, pudiera tener efectos nocivos sobre la salud a largo plazo.

Lo recién expuesto se ajusta al enfoque transaccional de Lazarus y Folkman (1986) y al modelo de mediación moderada propuesto por Guarino (2009). Estos autores indican que el esfuerzo adaptativo conductual, cognitivo y emocional- a los cambios del entorno se da en correspondencia con la percepción de éste como amenazante o desbordante de los recursos personales; y está moderado por las diferencias individuales presentes en el proceso de evaluación cognitiva, repercutiendo posteriormente en el estado de salud. 
Como consecuencia, puede pensarse que variables emocionales de personalidad presentes en la manera de relacionarse y de desempeñarse en el trabajo, jueguen un papel importante en el grado de estrés percibido y en el estado general de salud de los educadores. No obstante, en los institutos universitarios de Venezuela no se habían realizado estudios previos con el plantel hasta el momento docente, que pudieran tomarse como antecedentes y que arrojasen datos sobre las diferencias individuales asociadas al afrontamiento del estrés y su relación con la salud de los profesores.

Existe evidencia de que los estilos emocionales de afrontamiento del estrés están asociados a la aparición de síntomas físicos y psicológicos. Así mismo el afrontamiento por evitación acentúa los efectos negativos del estrés sobre la salud; mientras que el afrontamiento racional y por desapego emocional actúan como variables moderadoras protectoras de los síntomas físicos en diversas poblaciones (Aldana \& Guarino, 2012; Bethelmy \& Guarino, 2008a; Guarino, 2009; Guarino, Scremín \& Borrás, 2010; 2012; Herrera \& Guarino 2008; Sojo \& Guarino, 2011; Roger, 2007; Torres \& Guarino, 2013;). Falta comprobar si esta misma relación se presenta en el proceso de estrés de profesores universitarios.

La sensibilidad emocional es otra variable que puede moderar la relación estrés-salud de los docentes. Según Guarino (2005; 2009), el constructo define la alta disposición de los individuos a experimentar intensas emociones negativas frente a las demandas ambientales, así como la habilidad para reaccionar ante las emociones expresadas por otros; ésta última medida a través de las siguientes dimensiones:

- Sensibilidad egocéntrica negativa: tendencia de los individuos a reaccionar negativamente con emociones de aprehensión, rabia, desesperanza, vulnerabilidad y auto-crítica ante las demandas ambientales y situaciones estresantes.

- Sensibilidad interpersonal positiva: tendencia a orientarse emocionalmente hacia los otros, caracterizado por la habilidad de reconocer con facilidad las emociones de otros y mostrar preocupación y compasión por aquellos que sufren o pasan por momentos difíciles.

- Distanciamiento emocional: tendencia a evitar reacciones emocionales negativas de los otros, manteniéndose distantes de aquellos que puedan estar experimentando circunstancias difíciles.

Esta variable ha sido estudiada en diversas poblaciones como estudiantes universitarios (Guarino, 2005; Guarino \& Roger, 2005), médicos (Bethelmy
\& Guarino, 2008a, 2008b; Torres \& Guarino, 2013), cadetes (Herrera \& Guarino, 2008), mujeres embarazadas (Guarino, 2010; Guarino, Scremín \& Borrás, 2012) y profesionales de diversas disciplinas (Goncalves, Feldman \& Guarino, 2009). Al igual que el afrontamiento, el rol moderador de la sensibilidad emocional en la relación estrés-salud todavía no ha sido establecido en profesionales de la educación superior.

Finalmente, este estudio desea explorar el papel de la tolerancia a la incertidumbre, medida a través del ERI (ver Lousinha \& Guarino, 2010) en la relación estrés-salud de los docentes, basado en los estudios de Greco (Greco \& Roger, 2001; 2003) sobre los efectos nocivos de la incertidumbre emocional y cognitiva sobre la salud. La intolerancia constituye un esquema disfuncional que dirige la forma de procesar la información y contribuye al desarrollo y mantenimiento de la preocupación sin fundamento objetivo real. Así mismo, conlleva la percepción de dificultades donde efectivamente no las hay, lo cual repercute a posteriori de manera negativa en la salud. El constructo está conformado por tres dimensiones: Incertidumbre Emocional, concebida en términos de preocupación o ansiedad; Deseo de Cambio entendido como el deseo de aventurarse y realizar cambios; e Incertidumbre Cognitiva manifestada como la necesidad apremiante de planificar y tener certeza.

Los resultados arrojados por este estudio, no sólo podrían fortalecer los modelos teóricos propuestos sobre la moderación de las diferencias individuales en la relación estrés-salud de los individuos, sino que se constituirían en un aporte valioso para desarrollar programas de intervención dirigidos a modificar los factores de riesgo que actúen como moderadores del estrés en docentes universitarios.

\section{Método}

\section{Participantes}

La muestra estuvo constituída por 211 profesores universitarios provenientes de varias instituciones de educación superior de la ciudad de Caracas-Venezuela, 49,2\% hombres y 50,3\% mujeres, a quienes se les invitó a participar en la investigación a través de comunicaciones escritas, enviadas mediante los correos internos de sus respectivas instituciones. En estas comunicaciones, se les explicaba los objetivos del estudio y se solicitaba su previo consentimiento. En caso de firmar 
el consentimiento, los profesores eran invitados a completar el cuadernillo de cuestionarios y posteriormente a devolverlo a su departamento de adscripción, donde era recogido por las investigadoras.

\section{Instrumentos}

\section{- Escala de Sensibilidad Emocional (ESE)}

La escala mide la sensibilidad emocional en sus tres dimensiones, a saber, positiva, negativa y distanciamiento emocional. En la presente investigación se utilizó la versión en español de Guarino y Roger (2005) compuesta por 45 ítems. La mencionada escala permite obtener un puntaje acerca de la reactividad emocional de las personas, ubicándolas según sus características, en alguna de las dimensiones. La consistencia interna de la escala en la población hispanoparlante se considera satisfactoria; siendo $\alpha=0,824$ para el factor de sensibilidad egocéntrica negativa (SEN); $\alpha=0,742$ para el distanciamiento emocional (DE); y $\alpha=0,710$ para la sensibilidad interpersonal positiva (SIP). Las respuestas están reunidas en una escala de puntuación sumada, tipo Likert, con opciones de respuesta de verdadero o falso para cada uno de los ítems. Las personas que contestan verdadero a los ítems, reflejan la presencia de las características propias de cada uno de los componentes descritos.

\section{- Cuestionario de Estilos de Afrontamiento (CSQ)}

Para la medición de la variable estilos de afrontamiento, se utilizó la adaptación al español del Cuestionario de Estilos de Afrontamiento (CSQ) de Roger, Jarvis y Najarian (1993), desarrollada por Guarino, Sojo y Bethelmy (2007), la cual mide el afrontamiento en sus cuatro dimensiones: emocional, racional, evitación y desapego emocional. Esta última dimensión es una sub-escala de la dimensión emocional, entendida como la capacidad del individuo de ver las situaciones estresantes desde una perspectiva objetiva, sin involucrarse directamente con la experiencia demandante (ver Guarino, Sojo \& Bethelmy, 2007).

El instrumento está constituido por 40 ítems: el factor emocional está compuesto por 12 ítems, el racional está integrado de 10 ítems, el de desapego se compone de 13 ítems y el de evitación consta de 5 ítems. Los índices de confiabilidad de las dimensiones de la escala son $\alpha=0,78$ para afrontamiento emocional; $\alpha=0,65$ para afrontamiento por evitación; $\alpha=0,73$ para desapego emocional; y $\alpha=0,76$ para afrontamiento racional.

En este cuestionario se les pide a los participantes que ubiquen, en una escala de frecuencia (siempre, frecuentemente, algunas veces o nunca), la forma en que típicamente reaccionan frente a determinadas situaciones. La corrección del inventario se realiza sumando los valores que los participantes en la escala de frecuencia otorgaron a los ítems correspondientes a cada dimensión de la variable por separado. Los datos se interpretan en función de los descriptivos obtenidos. - Escala de Respuesta a la Incertidumbre (ERI)

La escala mide tres dimensiones del afrontamiento o la respuesta a la incertidumbre, a saber: incertidumbre emocional, deseo de cambio e incertidumbre cognitiva (ver Lousinha \& Guarino, 2010), adaptado de la Uncertainty Response Scale de Greco y Roger (2001). Ésta escala en concreto, está constituida por 45 ítems y cuatro opciones de respuesta que son: Siempre $(S)$, Frecuentemente (F), Algunas veces (A) y Nunca ( $N$. La dimensión de Deseo de Cambio está compuesta por 12 ítems y registró una consistencia interna de $\alpha=0.87$; la dimensión de Incertidumbre Emocional está compuesta por 15 ítems y una consistencia $\alpha=0.91$ y la dimensión de Incertidumbre Cognitiva contó con 18 ítems y un coeficiente de consistencia interna de $\alpha=0.87$.

\section{- Escala de Estrés Percibido (EEP)}

Escala de 10 ítems, versión traducida, adaptada y validada en español por Remor y Carrobles (2001), de la Perceived Stress Scale 10 items (PSS-10) de Cohen, Kamarck y Mermelstein (1983). Este es un instrumento de auto- reporte que evalúa el nivel de estrés percibido durante el último mes, determinando el grado de control que el individuo percibe haber tenido para manejar las experiencias estresantes. El formato de respuesta de la escala es de cinco puntos, en donde $0=$ Nunca; $1=$ Casi nunca; 2 = De vez en cuando; $3=$ A menudo; 4 = Muy a menudo. La consistencia interna oscila entre $\alpha=0.84$ y $\alpha=0.86$ en población hispana.

- Cuestionario de Salud General de Golberg (GHQ General Health Questionnaire)

Versión hispana de Molina y Andrade (2002). Esta escala está compuesta por 28 reactivos que describen síntomas de ansiedad, depresión, somatización y disfunción social, respecto a los cuales los participantes deben reportar la frecuencia relativa con la que los han padecido en las últimas semanas, con un rango de posibilidades desde $1=\mathrm{No}$, en lo absoluto , hasta $4=$ Mucho más que lo habitual (Goldberg, 1972; Molina \& Andrade, 2002). Las puntuaciones más altas reflejan una mejor salud. Los índices de consistencia interna (Alfa de Cronbach) resultaron óptimos para todas las dimensiones del GHQ en una muestra de 328 venezolanos, oscilando entre $\alpha=0,80$ y $\alpha=0,93$, con un $\alpha=$ 0,93 para la escala completa (Sojo, 2006). 


\section{Procedimiento}

Se envió una comunicación interna, circular en físico y/o vía mensajería interna, donde se informó al profesorado sobre la importancia del estudio y los beneficios que pudieran reportarles los resultados del mismo en términos de creación y aplicación de programas de prevención y promoción de salud. En la misiva se explicó de forma concisa en qué consistía la investigación y se adjuntó la carta de consentimiento para ser firmada por los docentes que accedieran a participar y los instrumentos de medición. Se les indicó que en caso afirmativo, completaran el cuadernillo de cuestionarios completo y lo entregaran a la secretaria de sus respectivos departamentos, para ser recogido posteriormente por las investigadoras. Las respuestas fueron absolutamente anónimas, dado que se consultaba únicamente el género, la edad, los años de servicio en la institución y la categoría académica. Se entregaron a las instituciones alrededor de 400 cuestionarios, aunque fueron devueltos completados sólo 21.

\section{Resultados}

Inicialmente se analizaron los datos descriptivos de las variables estudiadas, para conocer su comportamiento en la población. Cabe destacar que los puntajes totales de los individuos en las diferentes dimensiones, fueron ponderados para así facilitar su comparación dentro del mismo instrumento. La tabla 1 muestra los valores para la sensibilidad emocional en sus diversas dimensiones.

La sensibilidad egocéntrica negativa presenta una media de 0.23 , -la menor en contraste con las otras dos dimensiones- . Esto implica que los participantes

Tabla 1.

Estadísticos descriptivos para la sensibilidad emocional.

\begin{tabular}{lccc}
\hline & $\begin{array}{c}\text { Sensibilidad Ego Negativa } \\
(\text { SEN })\end{array}$ & $\begin{array}{c}\text { Distanciamiento Emocional } \\
(\mathrm{DE})\end{array}$ & $\begin{array}{c}\text { Sensibilidad Interpersonal Positiva } \\
\text { (SIP) }\end{array}$ \\
\hline Media & .2250 & .3769 & .5746 \\
Desv. típ. & .19262 & .27049 & .19231 \\
Mínimo posible & 0 & & .08 \\
Mínimo observado & .00 & .00 & \\
Máximo posible & 1 & & 1.00 \\
Máximo observado & .86 & 1.00 &
\end{tabular}

Tabla 2.

Estadísticos para las dimensiones de estilos de afrontamiento.

\begin{tabular}{lcccc}
\hline & $\begin{array}{c}\text { Afrontamiento Emocional } \\
(\text { EMO) }\end{array}$ & $\begin{array}{c}\text { Afrontamiento Racional } \\
(\text { RAC) }\end{array}$ & $\begin{array}{c}\text { Afrontamiento por } \\
\text { Desapego Emocional } \\
\text { (DES) }\end{array}$ & $\begin{array}{c}\text { Afrontamiento por } \\
\text { Evitación } \\
(\text { EVI) }\end{array}$ \\
\hline Media & 1.6999 & 3.0199 & 2.1330 & 2.0314 \\
Desv. típ. & .33351 & .42552 & .33280 & .58948 \\
Mínimo posible & 1.00 & & & \\
Mínimo observado & 1.00 & 1.91 & 1.08 & 1.00 \\
Máximo posible & 4.00 & & & 3.80 \\
Máximo observado & 2.91 & 4.00 & 3.31 & \\
\hline
\end{tabular}


Tabla 3.

Estadísticos para las dimensiones de tolerancia a la incertidumbre.

\begin{tabular}{lccc}
\hline & Deseo de Cambio & Incertidumbre Cognitiva & Incertidumbre Emocional \\
\hline Media & 2.0616 & 1.9630 & .7308 \\
Desv. típ. & .50986 & .40888 & .45082 \\
Mínimo posible & .00 & .00 & .00 \\
Mínimo observado & .58 & .82 & .00 \\
Máximo posible & 3.00 & 3.00 & 3.00 \\
Máximo observado & 3.00 & 2.94 & 2.87 \\
\hline
\end{tabular}

presentan bajos niveles en esta dimensión; mientras que proporcionalmente, muestran una mayor sensibilidad interpersonal positiva, lo cual sugiere una moderada capacidad para empatizar con las emociones de los otros.

La tabla 2 muestra los resultados para el afrontamiento al estrés y sus dimensiones.

Según se puede observar en los valores de la tabla, los docentes evaluados mostraron emplear de forma proporcionalmente más amplia el estilo de afrontamiento racional al lidiar con los eventos estresantes, a diferencia de las estrategias por desapego y evitación. En último lugar se observa el afrontamiento emocional, lo que sugiere un uso poco frecuente de esta forma de afrontamiento en la muestra.

La tabla 3 presenta los resultados para las dimensiones del ERI - Escala de Respuesta a la Incertidumbre.

Los resultados indican que los docentes evaluados mostraron mayormente un deseo de cambio frente a las situaciones inciertas; aunque con puntajes muy cercanos, mostraron una moderada incertidumbre cognitiva. La incertidumbre emocional mostró los valores más bajos de las tres dimensiones, lo que sugiere que esta forma de respuesta frente a las circunstancias inciertas es poco frecuente.

La tabla 4 muestra los descriptivos para el estrés percibido, indicando que los docentes reportaron un estrés moderadamente bajo, con respecto a los valores mínimo y máximo posibles. Esto es contrario a lo que se hubiera esperado en esta población.

Finalmente, en la tabla 5 se presentan los resultados para las cuatro dimensiones del cuestionario GHQ, que mide el estatus de salud física y psicológica de los evaluados. Es preciso señalar que una puntuación de 3 indica que la salud de la persona se ha mantenido estable en las últimas semanas, mientras que puntajes
Tabla 4.

Estadísticos para estrés percibido.

\begin{tabular}{lc}
\hline Media & 13.4592 \\
\hline Desv. típ. & 5.15238 \\
Mínimo posible & 0.00 \\
Mínimo observado & 2.00 \\
Máximo posible & 40.00 \\
Máximo observado & 30.00 \\
\hline
\end{tabular}

menores indican un deterioro de la salud del individuo en la respectiva dimensión.

Tal como se muestra en la tabla, las dimensiones en las que los docentes reportan mayor deterioro de la salud, son la disfunción social y la somatización respectivamente; mientras que reportan menos síntomas de ansiedad y aún menos síntomas de depresión. El puntaje total de la muestra para la salud global (ponderación de las cuatro dimensiones) fue de 2.44 , lo cual sugiere un estatus de salud moderadamente alto para este grupo evaluado.

Posteriormente, una vez comprobados los supuestos para calcular las ecuaciones de regresión jerárquica que permiten evaluar el rol moderador de las diferencias individuales en la relación estrés-salud, se llevaron a cabo las ecuaciones siguiendo las orientaciones de Baron y Kenny (1986). Para reducir el número de análisis efectuados, se empleó como variable dependiente en todas las ecuaciones, el puntaje de salud global arrojado por el cuestionario GHQ.

La tabla 6 presenta los resultados usando como variable predictora el Estrés Percibido y como moderadoras la Sensibilidad Emocional en sus tres dimensiones 
Tabla 5.

Estadísticos para las cuatro dimensiones de salud general.

\begin{tabular}{lcccc}
\hline & Somatización & Ansiedad & Disfunción Social & Depresión \\
\hline Media & 2.2850 & 2.4068 & 2.1509 & 2.9108 \\
Desv. típ. & .52154 & .55829 & .33587 & .22407 \\
Mínimo posible & 0.00 & 0.00 & 0.00 & 0.00 \\
Mínimo observado & .00 & .43 & 1.00 & 1.43 \\
Máximo posible & 3.00 & 3.00 & 3.00 & 3.00 \\
Máximo observado & 3.00 & 3.00 & 3.00 & 3.00 \\
\hline
\end{tabular}

SEN, DE, SIP. En el primer paso, se introdujo el Estrés Percibido como variable predictora. En el segundo paso, se agregaron las tres dimensiones (SEN, DE, SIP) de Sensibilidad Emocional por separado. Por último, en el tercer paso, se incorporaron los productos cruzados del Estrés Percibido por cada una de las dimensiones de Sensibilidad Emocional (Z31=EPxSEN; Z32=EPxDE; $\mathrm{Z} 33=\mathrm{EPxSIP})$.

De acuerdo a los resultados arrojados para el coeficiente de determinación corregido, se observa que a través del Estrés Percibido, paso 1, se puede explicar un $40 \%$ de la varianza en los puntajes de Salud General; al incorporar las dimensiones de SE en el paso 2, se logra explicar un $50.8 \%$ y en el paso 3 , una vez incorporados los productos cruzados (Z31, Z32 y Z33) se explica un $1.1 \%$ adicional. De lo anterior se evidencia que en los modelos 1 y 2 se producen cambios significativos en $\mathrm{F}$; sin embargo, en el modelo 3 dicho cambio no es significativo para el total de las interacciones. Observando los coeficientes estandarizados específicos para cada interacción, se evidencia que la sensibilidad egocéntrica negativa es la única que está actuando como moderadora de la relación estrés-salud en esta muestra, debido a que dicha interacción resulta significativa (ver tabla 7)

Lo anterior sugiere que a determinados niveles de estrés percibido, una mayor sensibilidad egocéntrica negativa incrementa el impacto de la tensión sobre la salud general de los docentes, convirtiéndose así en un factor de riesgo.

La tabla 8 muestra los resultados de la ecuación de regresión usando los estilos de afrontamiento como variable moderadora. Tal como se hizo en el análisis anterior, en el primer paso se introduce el Estrés Percibido, en el segundo paso se introducen los Estilos de Afrontamiento y en el tercer paso se incorporan los productos cruzados del Estrés Percibido con cada una de las dimensiones de Estilos de Afrontamiento (Z21=EPxEMO; Z22=EPxEVI; Z23=EPxDES; Z24=EPxRAC).

En cuanto al coeficiente de determinación corregido, los resultados muestran que el Estrés Percibido, explica un 39.9\% de la varianza de la Salud General. Al

Tabla 6.

Análisis de regresión jerárquica para estrés percibido por sensibilidad emocional.

\begin{tabular}{lccccc}
\hline & & & \multicolumn{3}{c}{ Estadísticos de Cambio } \\
\cline { 4 - 6 } Modelo & $\begin{array}{c}\text { R cuadrado } \\
\text { corregida }\end{array}$ & $\begin{array}{c}\text { Error típ. de } \\
\text { estimación }\end{array}$ & $\begin{array}{c}\text { Cambio } \\
\text { R cuadrado }\end{array}$ & Cambio enF & $\begin{array}{c}\text { Sig. } \\
\text { Cambio en F }\end{array}$ \\
\hline 1 & .400 & .24974 & .404 & 104.859 & .000 \\
2 & .508 & .22604 & .117 & 12.400 & .000 \\
3 & .519 & .22354 & .020 & 2.138. & 098 \\
\hline
\end{tabular}


Tabla 7.

Coeficientes Estandarizados en el análisis de regresión jerárquica para estrés percibido y sensibilidad emocional.

Coeficiente estandarizados

\begin{tabular}{|c|c|c|c|c|c|}
\hline \multicolumn{2}{|c|}{ Model } & \multirow[t]{2}{*}{$\mathrm{s}$} & \multirow{2}{*}{$\frac{\text { Beta }}{-.635}$} & \multirow{2}{*}{$\begin{array}{c}\mathrm{tS} \\
-10.240 .\end{array}$} & \multirow{2}{*}{$\frac{\text { ig }}{000}$} \\
\hline 1 & Estrés & & & & \\
\hline \multirow[t]{4}{*}{2} & Estrés & & -.444 & -6.800 & .000 \\
\hline & SEN & & -.426 & -5.901 & .000 \\
\hline & $\mathrm{DE}$ & & .081 & 1.204 & .230 \\
\hline & SIP & & .064 & 1.066 & .288 \\
\hline \multirow[t]{7}{*}{3} & Estrés & & -.424 & -6.441 & .000 \\
\hline & SEN & & -.358 & -4.614 & .000 \\
\hline & $\mathrm{DE}$ & & .058 & .845 & .399 \\
\hline & SIP & & .065 & 1.074 & .284 \\
\hline & z31 & & -.184 & -2.243 & .026 \\
\hline & z32 & & .105 & 1.331 & .185 \\
\hline & z33 & & -.024 & -. 375 & .708 \\
\hline
\end{tabular}

Tabla 8.

Análisis de regresión jerárquica para estrés percibido por estilos de afrontamiento.

\begin{tabular}{lccccc}
\hline Modelo & $\begin{array}{c}\text { R cuadrado } \\
\text { corregida }\end{array}$ & $\begin{array}{c}\text { Error típico de } \\
\text { estimación }\end{array}$ & $\begin{array}{c}\text { Cambio en R } \\
\text { cuadrado }\end{array}$ & Cambio en F & $\begin{array}{c}\text { Sig. de cambios } \\
\text { en F }\end{array}$ \\
\hline 1 & .399 & .2559 & .402 & 130.400 & .000 \\
2 & .447 & .2454 & .059 & 5.241 & .001 \\
3 & .479 & .2382 & .044 & 3.881 & .005 \\
\hline
\end{tabular}

incluir al incluir los Estilos de Afrontamiento se explica un $44.7 \%$. Por 'ultimo, al incorporar los productos cruzados (Z21, Z22, Z23 y Z24) se explica un $47.9 \%$ de la varianza de la Salud General.

En la tabla 9 se aprecian los resultados de los coeficientes estandarizados en el Análisis de Regresión Jerárquica para Estrés Percibido y Estilos de Afrontamiento.

Tal como se observa en los coeficientes estandarizados, una vez comprobados los efectos directos del estrés percibido y los estilos de afrontamiento sobre la salud global de los docentes, se tiene que la interacción Z21 (EPxEMO) evidencia un efecto moderador del Afrontamiento Emocional en la relación Estrés Percibido - Salud General. Esto es, a mayores niveles de estrés percibido y mayor uso de estilos de afrontamiento emocional, mayor será el impacto sobre la salud global de los docentes.

$\mathrm{EMO}=$ Estilo emocional, $\mathrm{RAC}=$ Estilo racional, DES= Estilos por desapego emocional, EVI= Estilo de evitación 
Tabla 9.

Coeficientes estandarizados en el análisis de regresión jerárquica para estrés percibido y estilos de afrontamiento.

\begin{tabular}{|c|c|c|c|c|}
\hline & & $\begin{array}{c}\text { Coeficientes } \\
\text { estandarizados }\end{array}$ & & \\
\hline & & Beta & $\mathrm{t}$ & Sig. \\
\hline \multirow[t]{2}{*}{1} & Estrés & -.634 & -11.423 & .000 \\
\hline & percibido & & & \\
\hline \multirow[t]{6}{*}{2} & Estrés & -.492 & -7.586 & .000 \\
\hline & percibido & & & \\
\hline & EMO & -.139 & -2.104 & .037 \\
\hline & RAC & .194 & 3.281 & .001 \\
\hline & DES & -.101 & -1.804 & .073 \\
\hline & EVI & -.060 & -1.038 & .301 \\
\hline \multirow[t]{10}{*}{3} & Estrés & -.454 & -6.900 & .000 \\
\hline & percibido & & & \\
\hline & EMO & -.068 & -1.013 & .313 \\
\hline & RAC & .197 & 3.364 & .001 \\
\hline & DES & -.079 & -1427 & .155 \\
\hline & EVI & -.076 & -1.301 & .195 \\
\hline & $\mathrm{z} 21$ & -.135 & -2.123 & .035 \\
\hline & $z 22$ & .099 & 1.682 & .094 \\
\hline & $\mathrm{z} 23$ & .062 & 1.138 & .257 \\
\hline & z24 & -.066 & -1.107 & .270 \\
\hline
\end{tabular}

Finalmente, la tabla 10 muestra los resultados de la ecuación de regresión usando los puntajes del cuestionario de tolerancia a la incertidumbre como variable moderadora. Se siguió el mismo procedimiento de análisis que con las anteriores ecuaciones jerárquicas.

De acuerdo a los resultados obtenidos para el coeficiente de determinación corregido, se observa que a través del Estrés Percibido (paso 1), se puede explicar un $45.7 \%$ de la varianza de Salud General. Al incorporar la Tolerancia a la Incertidumbre (paso 2), se explica un
$56.2 \%$. Y en el paso 3, una vez agregados los productos cruzados (Z12, Z11 y Z13) se explica un 56.5\%. De este modo, al revisar la significación se evidencia que en los modelos 1 y 2 se producen cambios significativos en $F$ $(\mathrm{p}=.000)$, en cambio para el modelo 3 el cambio no es significativo $(p=270)$. En base a estos resultados, no parece plausible la hipótesis de una posible moderación de la variable Tolerancia a la Incertidumbre, en sus tres dimensiones. Este hecho se verifica a través de la insignificancia de los coeficientes estandarizados para los productos de interacción. 
Tabla 10.

Análisis de regresión jerárquica para estrés percibido por tolerancia a la incertidumbre.

\begin{tabular}{lccccc}
\hline Modelo & $\begin{array}{c}\text { R cuadrado } \\
\text { corregida }\end{array}$ & $\begin{array}{c}\text { Error típico de } \\
\text { estimación }\end{array}$ & $\begin{array}{c}\text { Cambio en R } \\
\text { cuadrado }\end{array}$ & Cambio en F & $\begin{array}{c}\text { Sig. de cambios } \\
\text { en F }\end{array}$ \\
\hline 1 & .457 & .2442 & .461 & 127.395 & .000 \\
2 & .562 & .2193 & .113 & 12.869 & .000 \\
3 & .565 & .2186 & .011 & 1.321 & .270 \\
\hline
\end{tabular}

\section{Discusión}

El objetivo de la presente investigación consistió en estudiar el papel de ciertas diferencias individuales en la salud de una muestra profesoral a nivel de Educación Superior. Específicamente, se investigó el rol moderador de la Sensibilidad Emocional, la Tolerancia a la Incertidumbre y los Estilos de Afrontamiento, adoptados por los docentes universitarios en la relación entre su percepción de estrés y su salud general.

Contrariamente a lo esperado, los resultados indicaron que en general la muestra no se percibe con altos niveles de estrés, según lo arrojado por las puntuaciones de las medias en la escala para evaluar dicha variable. Haber obtenido datos que hablen de baja percepción de estrés pudiera causar asombro, sobre todo si se toman en cuenta las profundas transformaciones socio-educativas a que han estado sometidas en años recientes las casas de educación superior en Venezuela. Mencionamos especialmente los institutos y colegios universitarios; más ahora, en el marco de su conversión hacia universidades politécnicas que exigen un perfil docente orientado a la participación social. No obstante, estos resultados parecieran coincidir con lo reportado por Travers y Cooper (1997, en Crabay, 2001), quienes señalan que con frecuencia los profesores se resisten a reconocer hasta qué punto sufren de estrés, fundamentalmente por temor a un señalamiento de debilidad.

Con respecto a la variable salud global, los resultados indicaron un estatus de salud moderadamente bueno para esta muestra, lo cual también contrasta con los resultados esperados para una población como ésta, vulnerable y en riesgo. Tal vez podría considerarse que precisamente la presencia de un mayor afrontamiento racional y bajos niveles de estrés estarían protegiendo la salud de estos docentes frente a las demandas labores. Los resultados de esta investigación, de hecho, revelan que esta muestra de docentes emplea mayormente formas de afrontamiento racional. En este sentido, investigaciones previas habían determinado el impacto de esta forma de afrontamiento funcional al mejoramiento del impacto del estrés sobre la salud (Aldana \& Guarino 2012; Bethelmy \& Guarino, 2008a; Guarino, 2009; Herrera, 2006; Sojo \& Guarino, 2011; Torres \& Guarino, 2013).

La noción de que la muestra evaluada es relativamente sana y adaptada, se encuentra apoyada por los puntajes altos en sensibilidad interpersonal positiva y deseo de cambio frente a la incertidumbre. Las cuales se pueden considerar como características de personalidad, protectoras de la salud y la estabilidad emocional, tal como ha sido reseñado por Guarino $(2004 ; 2009)$ en sus investigaciones sobre la sensibilidad emocional, así como por Greco y Roger $(2001 ; 2003)$.

Aún más, un estudio reciente estableció que en el caso de los docentes venezolanos, pudiera estar actuando como variables protectoras o amortiguadoras de los efectos del estrés sobre el bienestar psicológico: la satisfacción laboral relacionada con la autonomía; el trabajo en sí mismo y las condiciones laborales (D’Aubeterre, 2011) De tal manera que la actividad docente puede ser percibida por el maestro como estimulante, si éste experimenta las condiciones mencionadas anteriormente.

Aun teniendo presentes los resultados anteriores, el objetivo central de esta investigación consistió en determinar si las diferencias individuales mencionadas, actuaban como moderadoras en la relación entre el estrés y la salud de los docentes, para lo cual se llevaron a cabo ecuaciones de regresión que permiten precisamente dar respuesta a estas interrogantes (Baron \& Kenny, 1986). Con ello podría entenderse cuáles son los factores específicos que determinan que unos docentes reporten problemas de salud y otros no, frente a las mismas demandas laborales.

Los resultados de este estudio apoyaron los hallazgos previos sobre el tema, en el sentido de que una mayor sensibilidad egocéntrica negativa y un mayor uso de afrontamiento emocional actúan como 
factores potenciadores de los efectos del estrés sobre la salud. De esta manera debe entenderse que, ante un nivel determinado de estrés percibido, el mostrar una mayor sensibilidad egocéntrica negativa, por un lado, y el emplear mayormente un estilo de afrontamiento emocional, por el otro, conducen a un mayor deterioro de la salud física y mental, concluyéndose que tales dimensiones de la sensibilidad emocional y el afrontamiento, respectivamente, actúan como moderadoras de la relación entre el estrés percibido y la salud. Si bien estas relaciones ya habían sido establecidas en otras poblaciones (Guarino, 2005; 2009; Bethelmy \& Guarino, 2008a; Sojo \& Guarino, 2011), aún no habían sido determinadas con grupos de docentes universitarios.

Específicamente con respecto a los estilos de afrontamiento, Griva y Joekes (2003) sugieren que el afrontamiento centrado en la emoción resulta menos efectivo para los docentes respecto al manejo de situaciones estresantes, puesto que puede impedir conductas adaptativas y propiciar comportamientos menos funcionales relacionados con la salud y la enfermedad. Por un lado, si el afrontamiento es pasivo-emocional, estará asociado con la depresión del sistema inmune, incrementando la vulnerabilidad a dolencias relacionadas con el sistema inmunológico; mientras que por otro lado, si lo que se produce es un afrontamiento activo con respuestas de fuerte expresión emocional, estará asociado a incrementos crónicos de la actividad periférica y central; y a la responsividad cardiovascular (Sandín, 1995, en Guerrero, 2003).

Por otro lado, la tolerancia a la incertidumbre en sus tres dimensiones, no mostró moderar la relación entre el estrés y la salud de los docentes; es decir, es independiente de la relación entre estos dos factores y no parece actuar ni amortiguando ni incrementando el impacto de la tensión sobre la salud general. Esto aún cuando la Incertidumbre Emocional contribuyó significativamente a la explicación de la Salud General en el modelo de ecuación, sugiriendo un efecto directo de esta dimensión sobre la variable criterio. Este último resultado apoya los hallazgos de Greco y Roger (2001; 2003) sobre el impacto negativo de esta forma de respuesta a la incertidumbre, en función de su asociación con reacciones fisiológicas importantes tanto hormonales como linfocitarias.

Investigaciones como la presente pretenden ir más allá del simple establecimiento de asociaciones entre el estrés percibido y su impacto sobre la salud, determinando en qué medida diversas variables de carácter personal o ambiental actúan como factores moduladores de tal relación, bien sea intensificando o amortiguando sus efectos. En este sentido, Hopwood (2007) refiere que el estudio de relaciones multivariables y específicamente, el establecimiento de las variables que median o moderan en los modelos explicativos, superan el paradigma de investigaciones previas. Así pues, este autor las denomina investigaciones de segunda generación, las cuales deben seguir desarrollándose con el objeto de establecer cada vez más, las interrelaciones complejas que existen en la experiencia individual del estrés.

Quedan por estudiarse muchas otras variables o factores personales que pudieran estar moderando la relación estrés-salud en diversas poblaciones y su determinación es fundamental para contar cada vez más con un modelo explicativo comprehensivo, cuyos resultados deriven en programas de intervención y control del estrés que abarquen todos los componentes implicados en el fenómeno, tal como lo sugieren Crespo y Labrador (2003). Por otro lado, conviene continuar explorándose el tema de la relación estrés-personalidad-salud, pero ahora desde una perspectiva más cualitativa, que permita entender a profundidad la complejidad de las relaciones entre estas variables y las implicaciones que la experiencia de estrés tiene sobre la vida cotidiana de los individuos.

Finalmente, es recomendable que en este tipo de estudios donde se evalúan indicadores de salud y adaptación, se pueda contar con medidas más objetivas de las variables contempladas (medidas fisiológicas, inmunológicas, entrevistas clínicas, etc.). De este modo, se reduciría el uso excesivo de instrumentos de autoreporte que pudieran introducir errores y sesgos por la vía de la deseabilidad social, factor que pude haberse constituido en una limitación del presente trabajo.

\section{Referencias}

Aldana, G. \& Guarino L. (2012). Sobrecarga, afrontamiento y salud en cuidadoras de pacientes con demencia tipo Alzheimer. Summa Psicológica UST, 9(1), 5-14.

Baron, R. M. \& Kenny, D.A. (1986). The moderator mediator variable distinction in social psychological research-conceptual strategic and statistical considerations. Journal of Personality and Social Psychology, 51(6), 1173 - 1182.

Bethelmy, L. \& Guarino L. (2008a). Afrontamiento y Sensibilidad Emocional como Moderadoras de la relación Estrés-Salud de Médicos Venezolanos. Summa Psicológica. UST, 5(2), 3-16.

Bethelmy, L. \& Guarino L. (2008b). Estrés Laboral, Afrontamiento, Sensibilidad Emocional y Salud en Médicos Venezolanos. Revista Colombiana de Psicología, 17, 43-58.

Cisneros, Y. \& Ramírez M. (2009). Prevalencia de enfermedades en trabajadores académicos de una universidad pública según seguro de gastos médicos. Salud de los trabajadores, 17(2), 121-131.

Cohen, S., Janicki-Deverts, D. \& Miller, G. (2007). Psycological Stress and Disease. Journal of the American Medical Association JAMA, 298(14), 1685-1687. 
Crabay, M. I., (2001). Entre la Salud y la Educación Superior: una aproximación a la situación laboral de los enseñantes. Ponencia en el I Congreso Internacional "Entre Educación y Salud" y Primer Encuentro Nacional del Instituto Dr. Domingo Cabred. Argentina.

Crespo, M., y Labrador, F. (2003). Estrés. Madrid: Editorial Síntesis.

D’Aubeterre, M. (2011). Bienestar psicológico, fuentes de estrés ocupacional y satisfacción laboral en docentes de educación básica. (Tesis inédita de maestría). Universidad Simón Bolívar. Caracas, Venezuela.

García, E. (1996). Estrés, Profesión Docente y Personalidad del Docente. Ansiedad y Estrés, 2(2-3), 245-260.

Goncalves, L.; Feldman, L. \& Guarino, L. (2009). Estrés Laboral, Sensibilidad Egocéntrica Negativa y salud en profesionales venezolanos. Summa Psicológica UST, 6(1), 3-14.

González, N. (2008). Prevalencia del estrés en la satisfacción laboral de los docentes universitarios. Revista Electrónica de Humanidades, Educación y Comunicación Social REDHECS, 4(3), 68-89.

Greco, V. \& Roger, D. (2001). Coping with uncertainty: The construction and validation of a new measure. Personality and Individual Differences, 31, 519-534.

Greco, V. \& Roger, D. (2003). Uncertainty, stress and health. Personality and Individual Differences, 34, 1057-1068.

Griva, K. \& Joekes, K. (2003). UK Teachers Under Stress: Can we predict wellness on the basis of the characteristics of the teaching job?. Psychology and Health, 18(4), 457-471.

Guarino, L. (2004). Emotional Sensitivity: a new measure of emotional lability and its moderating role in the stress-illness relationship. (Tesis inédita de Doctorado), Universidad de York, York, Inglaterra.

Guarino, L. (2005). Sensibilidad Emocional: Su Rol Moderador en la Relación Estrés-Enfermedad. Psicología Conductual, 13(2), 217-230.

Guarino, L. (2009). Validación del modelo de sensibilidad emocional. Propuesta de un modelo de mediación moderada. Manuscrito sin publicar. Universidad Simón Bolívar. Caracas, Venezuela.

Guarino, L. (2010). Sensibilidad emocional, afrontamiento, salud y calidad de vida percibida en embarazadas venezolanas. Psicología y Salud, 20(2), 179-188.

Guarino, L. \& Roger, D. (2005). Construcción y validación de la Escala de Sensibilidad Emocional (ESE). Un nuevo enfoque para medir neuroticismo. Psicothema, 17(3), 465-470.

Guarino, L., Scremín, F., \& Borrás, S. (2010). Estilo emocional, salud y calidad de vida percibida en embarazadas. Summa Psicológica UST, 7(1), 79-90.

Guarino, L., Scremín, F., \& Borrás, S. (2012). Diferencias individuales como predictoras de la salud y calidad de vida percibida en embarazadas. Pensamiento Psicológico, 10(2), 135-148.

Guarino, L, Sojo V. \& Bethelmy, L. (2007). Adaptación y validación preliminar de la versión hispana del cuestionario de estilos de
Afrontamiento. (Coping Style Questionnaire, CSQ). Psicología Conductual, 15(2), 173 - 189.

Guerrero, E. (2003). Análisis pormenorizado de los grados de Burnout y afrontamiento del estrés docente en profesorado universitario. Anales de Psicología, 19(1), 145-158

Guil, A. (1998). El Estrés del Profesorado: Estrategias de Apoyo Social. Boletín de la Sociedad Española de Psicoterapia y Técnicas de Grupo, 96-103.

Herrera, V. (2006). Sensibilidad emocional y afrontamiento como moderadoras de la relación estrés-salud en cadetes. (Tesis inédita de maestría). Universidad Simón Bolívar, Caracas, Venezuela.

Herrera, V. \& Guarino, L. (2008). Personalidad, estrés y salud en cadetes navales venezolanos. Universitas Psicológica, 7(1), 185-198.

Hopwood, Ch. (2007). Moderation and mediation in Structural Equation Modeling: Applications for early intervention research. Journal of Early Intervention, 29(3), 262-272.

Lazarus, R. \& Folkman, S. (1986). Estrés y Procesos Cognitivos. Barcelona: Martínez Roca.

Lousinha, A., \& Guarino, L. (2010). Adaptación hispana y validación de la escala de respuesta a la incertidumbre. Pensamiento Psicológico, 8(15), 89-100.

Molina, J. \& Andrade, C. (2002). La estructura factorial del GHQ-60 en una muestra de población general: una versión escalar para población española. Revista Internacional On-line, 1(2). Recuperado de http//www.bibliopsiquis.com/asmr/0102/0102lef.htm.

Ng, D. \& Jeffery, R. (2003). Relationships between perceived stress and health behaviours in a sample of working adults. Health Psychology, 22(6), 638-642.

Remor, E. \& Carrobles J. A. (2001). Versión española de la escala de estrés percibido (PSS-14): Estudio psicométrico en una muestra VIH+. Ansiedad y Estrés, 7(2-3), 195-201.

Roger, D. (2007). Self-esteem, Stress and Emotion. Elseviewer, 3, 412-416

Sánchez, M. \& Maldonado, L. (2003) Estrés en docentes universitarios. Caso LUZ, URBE Y UNICA. Revista de Ciencias Sociales. 11(2), 323-335.

Steptoe, A. (1991). The links between stress and illness. Psychosomatic Research, 35, 633-644.

Sojo, V. \& Guarino, L. (2011). Mediated Moderation or Moderated Mediation: Relationship between Length of Unemployment, Resilience, Coping and Health. Spanish Journal of Psychology, $14,267-276$.

Tomé, M. \& Urdiales M. (1996). El estrés del profesor universitario. Ansiedad y Estrés, 2(2-3), 261-282.

Torres, B. \& Guarino, L. (2013). Diferencias individuales y burnout en médicos oncólogos venezolanos. Universitas Psychologica, 12(1), 95-104. 